\title{
Smoking as a Cofactor for Causation of Chronic Pancreatitis
}

\author{
A Meta-Analysis \\ Angelo Andriulli, MD, Edoardo Botteri, MSc, $\dagger$ Piero L. Almasio, MD, + Italo Vantini, MD, $\S$
Generoso Uomo, MD,// and Patrick Maisonneuve, Eng, $\dagger$
on behalf of an ad hoc committee of the Italian Association for the Study of the Pancreas
}

\begin{abstract}
Objectives: To assess the evidence for tobacco smoking as a risk factor for the causation of chronic pancreatitis.

Methods: We performed a meta-analysis with random-effects models to estimate pooled relative risks (RRs) of chronic pancreatitis for current, former, and ever smokers, in comparison to never smokers. We also performed dose-response, heterogeneity, publication bias, and sensitivity analyses.

Results: Ten case-control studies and 2 cohort studies that evaluated, overall, 1705 patients with chronic pancreatitis satisfied the inclusion criteria. When contrasted to never smokers, the pooled risk estimates for current smokers was 2.8 (95\% confidence interval [CI], 1.8-4.2) overall and 2.5 (95\% CI, 1.3-4.6) when data were adjusted for alcohol consumption. A dose-response effect of tobacco use on the risk was ascertained: the RR for subjects smoking less than 1 pack per day was 2.4 (95\% CI, 0.9-6.6) and increased to 3.3 (95\% CI, 1.4-7.9) in those smoking 1 or more packs per day. The risk diminished significantly after smoking cessation, as the RR estimate for former smokers dropped to a value of 1.4 (95\% CI, 1.1-1.9).

Conclusions: Tobacco smoking may enhance the risk of developing chronic pancreatitis. Recommendation for smoking cessation, besides alcohol abstinence, should be incorporated in the management of patients with chronic pancreatitis.
\end{abstract}

Key Words: chronic pancreatitis, smoking, cigarette, nicotine, alcohol, meta-analysis

(Pancreas 2010;39: 1205-1210)

$\mathrm{D}$ uring the past 2 decades, several experimental evidences indicated that cigarette/nicotine smoking induces marked pathological and functional changes in the exocrine pancreas. ${ }^{1}$ In humans, serum pancreatic enzymes after intravenous secretin injection increase substantially in smokers, but not in nonsmokers. ${ }^{2}$ Mechanisms by which nicotine induces such changes are mediated via signal transduction pathways in the pancreatic acinar cell, leading to enhanced levels of intracellular calcium release ${ }^{1}$ and/or impairment of pancreatic blood flow. ${ }^{3}$ Yet, only few pancreatic specialists advise alcoholic patients with chronic

From the *Division of Gastroenterology, "Casa Sollievo Sofferenza" Hospital, IRCCS, San Giovanni Rotondo; $†$ Division of Epidemiology and Biostatistics, European Institute of Oncology, Milan; \$Gastroenterology Unit, University of Palermo, Palermo; §Gastroenterology Unit, University of Verona, Verona; and ||Department of Internal Medicine, "A. Cardarelli" Hospital, Naples, Italy.

Received for publication November 17, 2009; accepted March 11, 2010.

Reprints: Angelo Andriulli, MD, Division of Gastroenterology, "Casa

Sollievo Sofferenza" Hospital, IRCCS, viale Cappuccini 1, 71013 San

Giovanni Rotondo, Italy (e-mail: a.andriulli@operapadrepio.it).

The authors declare no competing interests.

Writing assistance: None.

Copyright (C) 2010 by Lippincott Williams \& Wilkins pancreatitis for smoking cessation treatment, besides alcohol abstinence.

Cigarette smoking has been suggested as the single most important factor for the development of pancreatic cancer, causing a $75 \%$ increase in the risk compared with nonsmoking. ${ }^{4}$ In contrast, epidemiological data linking cigarette smoking to the development of chronic pancreatitis offer conflicting conclusions. ${ }^{5-20}$ Most studies indicate smoking as one of the candidate susceptibility factors for pancreatitis, $,-7,10-17,20$ whereas others have denied such an association. ${ }^{8,9,18}$ The close interaction between tobacco consumption and alcohol intake ${ }^{21}$ with heavy drinkers often being heavy smokers ${ }^{22,23}$ renders problematic to ascertain the relative pathogenic role of the 2 factors in tissue injury. Moreover, methodological differences among existing epidemiological studies cast doubt on the findings in this topic. Many of the positive reports have been confounded by the retrospective nature of data collection, which impacts significantly on the ability to accurately assess alcohol intake or cigarette-smoke exposure. ${ }^{24}$ Furthermore, the lack of appropriate controls is crucial for any study examining individual susceptibility to alcoholic pancreatitis: it has been argued that the best comparison for such studies must be between alcoholics with the disease and alcoholics without the disease so that the index and the control groups differ only in 1 variable, that is, the presence or absence of pancreatitis. ${ }^{8,25}$ Unfortunately, numerous studies in the literature have used only the general population as controls, making the interpretation of results controversial. $7,12,14,19,20$

The current review focuses on important epidemiological observation linking tobacco smoking to chronic pancreatitis development and provides insightful interpretation of what is known. The aims of this study were to provide a statistical evaluation using a meta-analytic approach of the strength of the association between tobacco smoking and chronic pancreatitis and to assess whether the association depends on study characteristics such as the control population, study designs, or level of exposure.

\section{MATERIALS AND METHODS}

\section{Search Strategy, Inclusion Criteria, and Data Abstraction}

We searched PubMed from 1966 to July 15, 2009, for the following terms: ([smoke OR cigarette OR tobacco OR smoking] AND [(chronic pancreatitis OR alcoholic chronic pancreatitis OR pancreatitis)]. Our search was restricted to human studies published in English language. We searched the reference lists of articles and reviews on the topic to identify additional relevant studies.

Only reports complying with the following inclusion criteria were included in the meta-analysis. First, studies should 


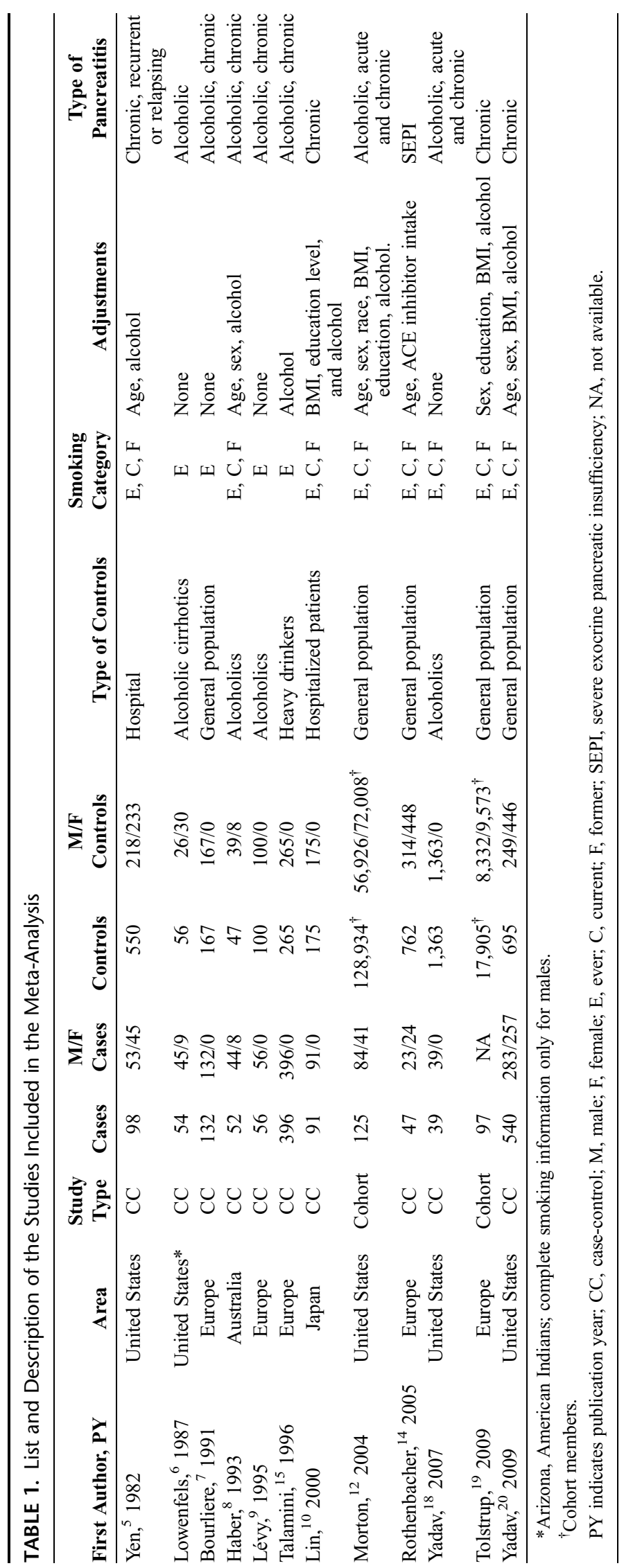

1206 | www.pancreasjournal.com 
contain the minimum information necessary to estimate the relative risk (RR) associated with tobacco smoking and a corresponding measure of uncertainty (ie, $95 \%$ confidence interval [CI], SE, or $P$ value of the significance of the estimate). Comparisons of interest were current versus never smokers, former versus never smokers, and ever versus never smokers. Second, studies should be independent: in case of multiple reports on the same population, we considered the estimates from the most informative report. When available, we used adjusted estimates. Three of us (A.A., P.L.A., and E.B.) independently abstracted the data, and we resolved discrepancies by committee.

\section{Data Analysis}

The RR was used as a measure of the association between cigarette smoking and pancreatitis. For case-control studies, odds ratios were used as estimates of the RR. We used randomeffects models to estimate pooled RRs. Homogeneity of effects across studies was assessed using the $\chi^{2}$ statistic ${ }^{26,27}$ and the $\mathrm{I}^{2}$ statistic, ${ }^{28}$ which represents the percentage of total variation across studies that is attributable to heterogeneity rather than to chance. Subgroup analyses and metaregression models were carried out to investigate potential sources of between-study heterogeneity, such as sex or study design. When several risk estimates were present in a single study (ie, separate estimates for men and women), we adjusted the pooled estimates for intrastudy (or within-study) correlation. ${ }^{27}$ Publication bias was evaluated by funnel plots and quantified by the Egger's test. ${ }^{29}$ Analyses were performed with the SAS software (version 8.2; SAS, Cary, NC). All $P$ values were 2-sided.

\section{RESULTS}

\section{Study Identification and Selection}

Our search returned 253 citations. Title and abstract review resulted in exclusion of 237 studies. This left 16 publications on the topic of interest, ${ }^{5-20}$ and full text was retrieved for detailed evaluation. Four studies ${ }^{11,13,16,17}$ were excluded because of multiple reports on the same setting of patients, ${ }^{16,17}$ of pooling in a single category former smokers and never smokers, ${ }^{11}$ or because the comparison group included patients with mild form of alcoholic chronic pancreatitis and moderate smokers were aggregated with never smokers as the reference group. ${ }^{13}$ We included in the meta-analysis 12 studies published between 1982 and $2009,{ }^{5-10,12,14,15,18-20}$ involving a total of 1705 chronic pancreatitis patients. The number of patients in each study ranged from 39 to 540 . Overall, 1235 (72\%) were males. Table 1 shows selected traits of the total study population and of the chronic pancreatitis cases.

\section{Results From the Meta-Analysis}

Results are outlined in Table 2 and illustrated as forest plots in Figures 1 to 3 . When contrasted to never smokers, pooled risk estimates were $2.8(95 \% \mathrm{CI}, 1.7-4.8)$ for ever smokers based on 12 studies, 2.8 (95\% CI, 1.8-4.2) for current smokers based on 8 studies, and 1.4 (95\% CI, 1.1-1.9) for former smokers based on 8 studies. We found evidence of heterogeneity among the studies for ever smokers $\left(P<0.01\right.$ and $\mathrm{I}^{2} 72 \%$ for all estimates; Table 2$)$ but not for current and former smokers. Risk estimates for ever smokers maintained some heterogeneity even in the subgroup analyses. The meta-analytical results did not differ when casecontrol studies and cohort studies were analyzed separately for each of the 3 smoking categories. When limiting the analysis to studies that controlled for alcohol consumption or included only alcoholics without chronic pancreatitis among controls, the alcohol-adjusted RR estimates were still significant despite the lower number of studies providing such data: compared with

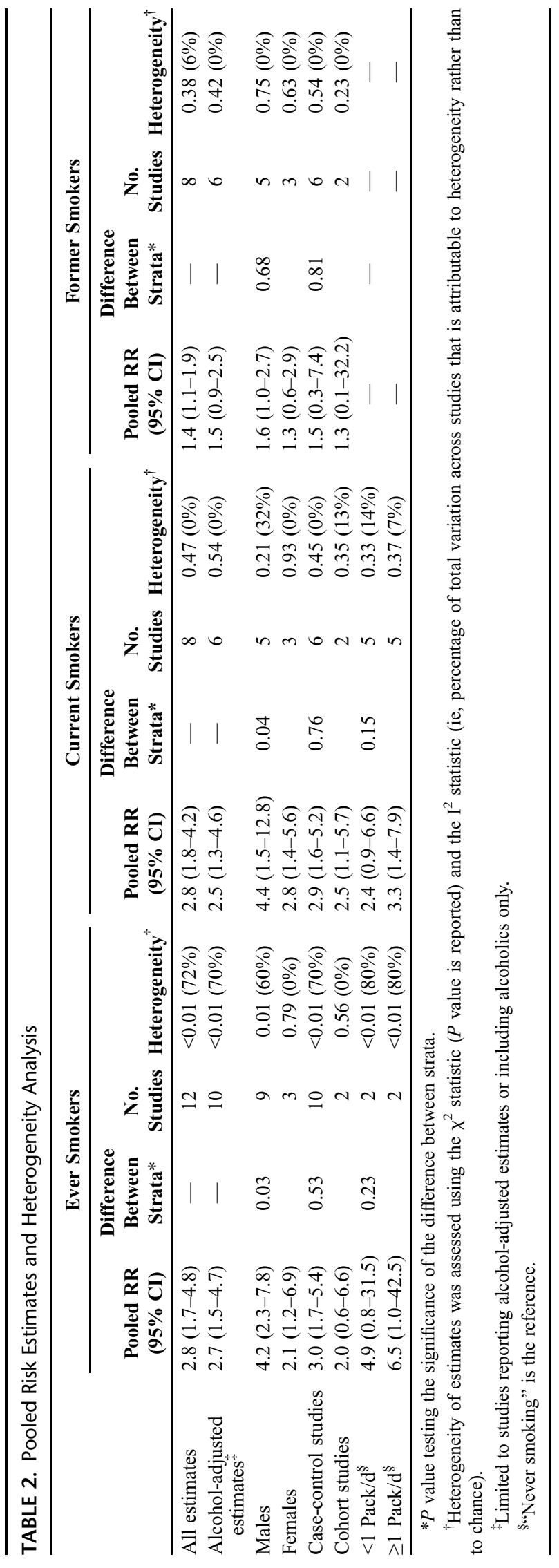

www.pancreasjournal.com 


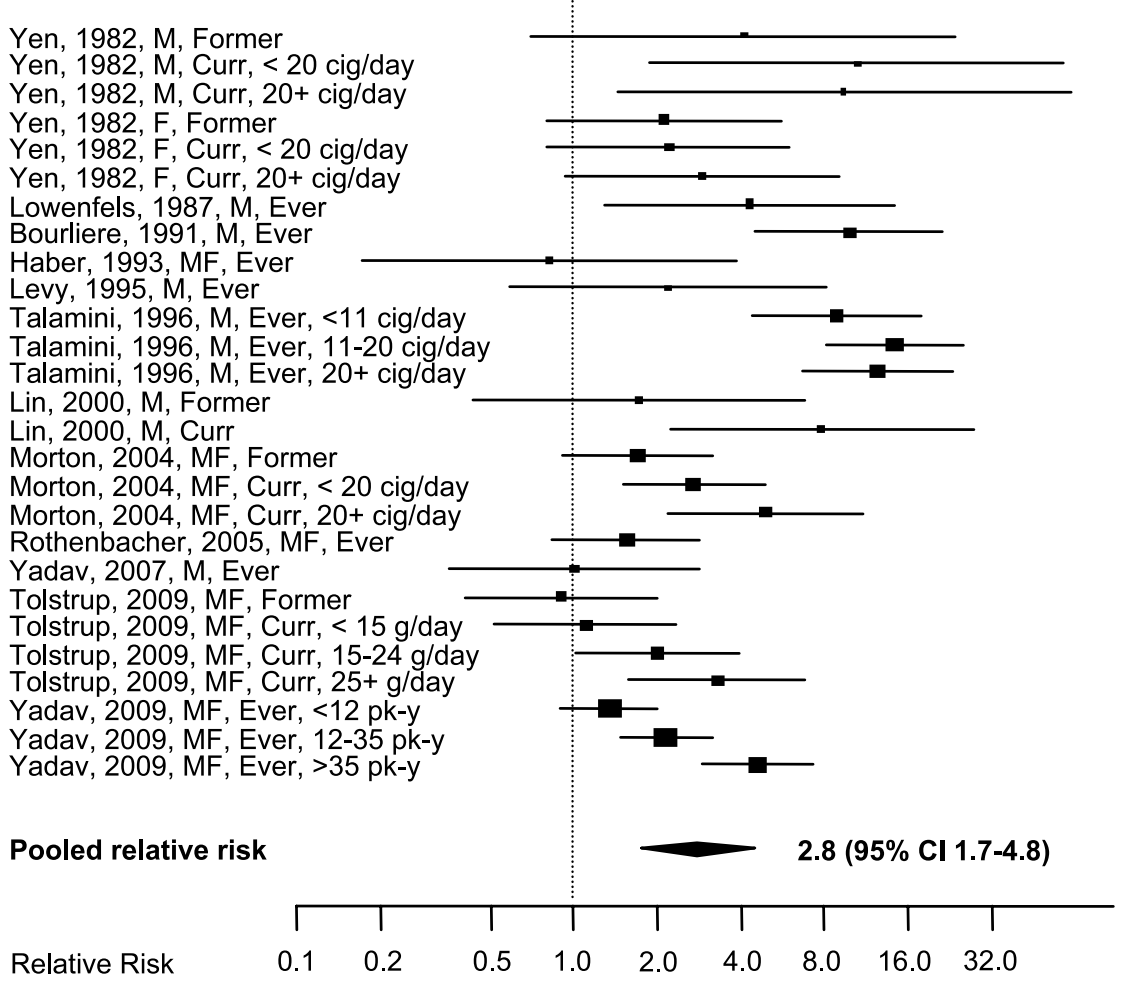

FIGURE 1. Forest plot of the RR of developing chronic pancreatitis for ever smokers versus never smokers. The size of each square is proportional to the study's weight (inverse of variance). Studies are sorted by publication year and identified by, from left to right: first author, publication year, sex (M indicates males; F, females; MF, males and females), category of exposure (ever, current, or former), and amount of exposure. When we limited the analysis to studies reporting alcohol-adjusted estimates (excluding Rothenbacher ${ }^{14}$ and Bourliere $\left.{ }^{7}\right)$, we obtained a pooled estimate of $2.7(95 \% \mathrm{Cl}, 1.5-4.7)$.

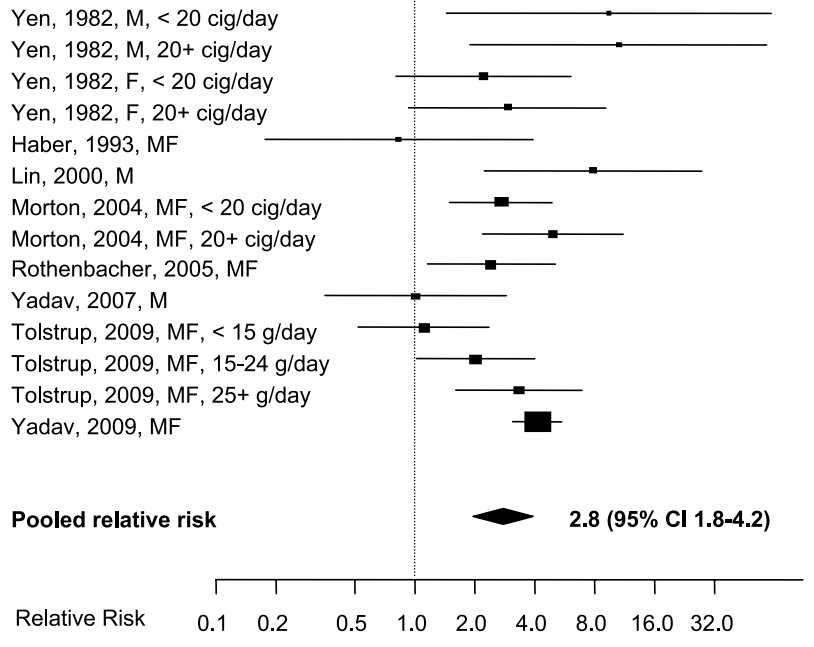

FIGURE 2. Forest plot of the RR of developing chronic pancreatitis for current smokers versus never smokers. The size of each square is proportional to the study's weight (inverse of variance). Studies are sorted by publication year and identified by, from left to right: first author, publication year, sex ( $\mathrm{M}$ indicates males; F, females; MF, males and females), and, where required, amount of exposure. When we limited the analysis to studies reporting alcohol-adjusted estimates (excluding Rothenbacher ${ }^{14}$ and $\left.\mathrm{Yadav}^{20}\right)$, we obtained a pooled estimate of $2.5(95 \% \mathrm{Cl}, 1.3-4.6)$.

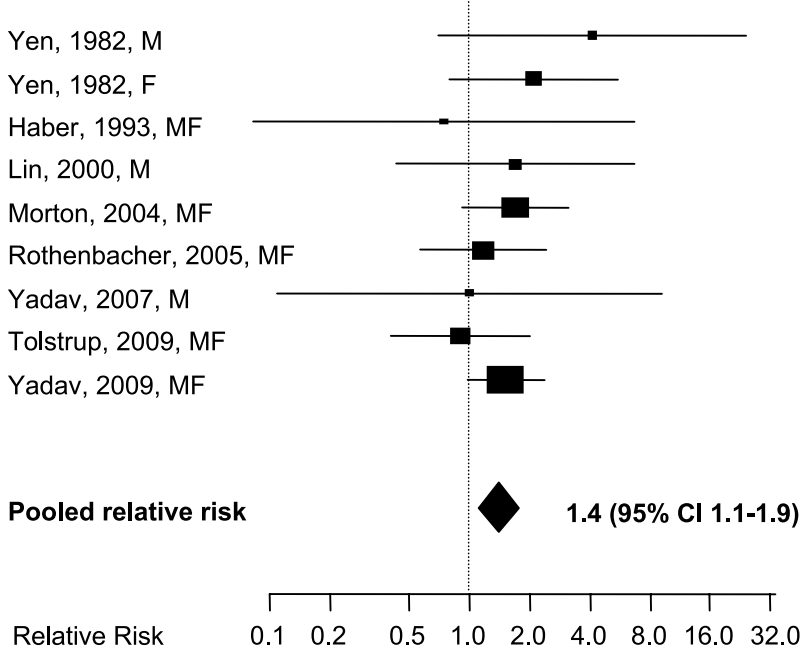

FIGURE 3. Forest plot of the RR of developing chronic pancreatitis for former smokers versus never smokers. The size of each square is proportional to the study's weight (inverse of variance). Studies are sorted by publication year and identified by, from left to right: first author, publication year and sex ( $M$ indicates males; F, females; MF, males and females). When we limited the analysis to studies reporting alcohol-adjusted estimates (excluding Rothenbacher ${ }^{14}$ and Yadav $^{20}$ ), we obtained a pooled estimate of 1.5 (95\% Cl, 0.9-2.5). 
never smokers, the RR estimates were 2.7 (95\% CI, 1.5-4.7) for ever smokers, 2.5 (95\% CI, 1.3-4.6) for current smokers, and 1.5 (95\% CI, 0.9-2.5) for former smokers.

Five studies $5,10,12,18,19$ reported stratification of patients for the amount of tobacco use. Among current smokers, we found a dose-response relationship between the amount of tobacco smoked and the risk of developing chronic pancreatitis: the RR for patients smoking less than 1 pack per day was 2.4 (95\% CI, 0.9-6.6) and increased to 3.3 (95\% CI, 1.4-7.9) in those smoking 1 or more packs per day. Analysis by funnel plots and Egger's test suggested that publication bias was not a problem in the context of our study (results not shown).

As alcoholic chronic pancreatitis is predominantly a male disease, ${ }^{30,31}$ we performed a subset analysis to clarify whether the risk was higher among male patients. We found significantly raised RR estimates for ever and current smokers in comparison to never smokers: RR of 4.2 (95\% CI, 2.3-7.8) and RR of 4.4 (95 CI, 1.5-12.8) based, respectively, on 9 and 5 studies; the association lost statistical significance for former smokers (RR, 1.6; 95\% CI, 1.0-2.7) based on 5 studies.

\section{DISCUSSION}

Smoking is an established risk factor for many forms of cancer. ${ }^{32}$ In particular, tobacco use approximately doubles the risk of pancreatic cancer ${ }^{4,32,33}$ and has been already identified as a risk factor for the susceptibility to chronic pancreatitis in addition to alcohol abuse. ${ }^{5-20}$ Yet, only few physicians advice smoking cessation when caring for either patients with alcohol addiction or those with an established chronic pancreatitis secondary to alcohol abuse. Plausible explanation for this paradox relates to either the inconsistency in the reported association with some negative reports ${ }^{8,14,18}$ and to the confounding effect of alcohol consumption, as cigarette smoking is often an inseparable addiction in most alcoholics. ${ }^{21-23}$ Therefore, it has been hard to separate the role of smoking from the effect of alcohol in causation of chronic pancreatitis.

The current meta-analysis supports and strengthens the evidence on the association between cigarette smoking and the risk for chronic pancreatitis: our analysis based on 12 independent studies has shown that, overall, tobacco use confers a 2- to 3-fold increased risk of developing a chronic pancreatitis in comparison to alcoholics or population-based controls who never smoked. Moreover, the likelihood of developing chronic pancreatitis was found to be proportional to the amount of tobacco use, suggesting that smoking exerts a dose-related effect on initiating pancreatic injury. Indeed, the pooled RR was $3.3(95 \%$ CI, 1.4-7.9) for smokers of 1 or more packs per day and was lower in those smoking less than 1 pack per day (RR, 2.4; 95\% CI, 0.9-6.6). Of note, the strength of the evidence is much stronger than the reported estimates for smoking as a risk factor for pancreatic adenocarcinoma. 4,32,33 The validity of the relationship between smoking and chronic pancreatitis is further highlighted by the lower risk after smoking cessation: compared with never smokers, the risk in current smokers seemed higher than in former smokers, with respective pooled RR estimates of $2.8(95 \% \mathrm{CI}, 1.8-4.2)$ and 1.4 (95\% CI, 1.1-1.9). Risk estimates for ever smokers were heterogeneous, whereas for current and former smokers they were not. This could be due to the fact that the category of ever smokers could contain, by definition, a great variety of types of smokers, and often the studies did not supply the details of exposure.

A strong argument against the role of tobacco smoking in the initiation of alcohol-induced injury to the pancreatic pa- renchyma was raised by Haber et $a 1,{ }^{8}$ who argued that the presence of 2 known factors, that is, alcohol and tobacco, would render difficult to ascertain the relative pathological contribution of either factor in tissue damage. A major strength of our study is to have controlled the RR estimates for alcohol consumption. In the event alcohol consumption might act as the sole or the predominant factor in the causation of chronic pancreatitis, one would expect that the risk observed for smoking weakens. On the contrary, we observed no variation in the magnitude of the risk estimates when we limited the analysis to the 10 studies $^{5,6,8-10,12,15,18-20}$ reporting alcohol-adjusted RR estimates or presenting data in alcoholics only. Although the risk was higher for current than for former smokers, the increasing risk with increasing levels of tobacco consumption and the stability of the risk estimates after adjustment for alcohol provide strong support for the role of tobacco smoking in the development of alcoholic chronic pancreatitis; it is still possible that residual confounding with alcohol exists and accounts for part of the association observed with tobacco smoking.

The comparisons between subjects with and without chronic pancreatitis would be expected to differ markedly from comparisons using controls drawn from the general population or subjects hospitalized for reasons other than those related to alcohol or smoking. Indeed, RR did not differ when controls were extracted from the general, healthy population or from hospitalized patients or when only alcoholics were included among controls.

Other issues concerning our analysis merit consideration. Most cases enrolled in the original, selected studies were patients with chronic pancreatitis, but a few cases of acute, relapsing/recurrent pancreatitis were also included in some studies ${ }^{5,12,18}$ and could have biased the association. In fact, much less evidence exists on the effect of tobacco smoking on acute or recurrent pancreatitis. Whereas recently Lindkvist et $\mathrm{al}^{34}$ found that smoking was associated with the incidence of acute pancreatitis in a dose-response manner, Pelli et a ${ }^{35}$ did not find smoking to be a risk factor for recurrent episodes after the first attack of acute alcoholic pancreatitis.

Although the number of studies included in this metaanalysis is limited and the study population characteristics and the assessment of the exposure to tobacco and alcohol often inadequate to obtain confident risk estimates, a series of studies provide further support to the role of tobacco smoking on pancreatic damage in patients with chronic pancreatitis. Among patients with chronic alcoholic and idiopathic pancreatitis, tobacco smoking seems to be associated with worsening of the disease, accelerating in particular the development of calcifications. ${ }^{24,36,37}$ Among patients with hereditary pancreatitis who face an elevated risk of being diagnosed with pancreatic adenocarcinoma, smoking seems to advance the age of cancer diagnosis by approximately 2 decades. ${ }^{38}$ Although pancreas cancer risk is not associated with one of the specific genetic mutations leading to hereditary pancreatitis, ${ }^{39}$ it seems to be related to the longstanding inflammation of the pancreas in these patients and be exacerbated by exposure to tobacco smoke.

Although a meta-analysis represents a powerful instrument to resolve eventually conflicting research results, increasing the effective sample size by pooling many studies and offering the possibility to explore sources of heterogeneity, it, however, suffers from the quality of the data analyzed. We recognize that the current meta-analysis is based on a series of reports in which alcohol and smoking history were poorly recorded, but in our view, it should be the basis for the planning of future research, using more objective measures of lifetime alcohol and tobacco consumption. 
In conclusion, the present investigation overcomes some of the limitations that may have hindered the detection of cigarette smoking as a trigger factor for chronic pancreatitis in previous studies. We have shown that cigarette smoke approximately doubles the risk of developing chronic pancreatitis, with a clear dose-response relationship with the number of cigarettes smoked per day. The association was still present after adjustment for alcohol consumption, and the risk was substantially lower among former than current smokers. The evidence presented offers solid backing for changing the current standard of care of alcoholic pancreatitis that includes the rehabilitation for alcoholism but not cessation of smoking. It may also serve for planning programs for the primary prevention of chronic pancreatitis by a combined effort directed toward abstaining not only from alcohol, but also from smoking.

\section{REFERENCES}

1. Chowdhury P, MacLeod S, Udupa KB, et al. Pathophysiological effects of nicotine on the pancreas: an update. Exp Biol Med. 2002;227:445-454.

2. Andriulli A, Masoero G, Amato A, et al. Serum immunoreactive cationic trypsinogen response to secretin in normal subjects. Am J Gastroenterol. 1983;78:579-583.

3. Hartwig W, Werner J, Ryschich E, et al. Cigarette smoke enhances ethanol-induced pancreatic injury. Pancreas. 2000;21:272-278.

4. Iodice S, Gandini S, Maisonneuve P, et al. Tobacco and the risk of pancreatic cancer: a review and meta-analysis. Langenbecks Arch Surg. 2008;393:535-545.

5. Yen S, Hsieh CC, MacMahon B. Consumption of alcohol and tobacco and other risk factors for pancreatitis. Am J Epidemiol. 1982;116:407-414.

6. Lowenfels AB, Zwemer FL, Jhangiani S, et al. Pancreatitis in a native American Indian population. Pancreas. 1987;2:694-697.

7. Bourliere M, Barthet $\mathrm{M}$, Berthezene $P$, et al. Is tobacco a risk factor for chronic pancreatitis and alcoholic cirrhosis? Gut. 1991;32:1392-1295.

8. Haber PS, Wilson JS, Pirola RC. Smoking and alcoholic pancreatitis. Pancreas. 1993;8:568-572.

9. Lévy P, Mathurin P, Roqueplo A, et al. A multidimensional case-control study of dietary, alcohol, and tobacco habits in alcoholic men with chronic pancreatitis. Pancreas. 1995; 10:231-238.

10. Lin Y, Tamakoshi A, Hayakawa T, et al. Cigarette smoking as a risk factor for chronic pancreatitis: a case-control study in Japan. Research Committee on Intractable Pancreatic Diseases. Pancreas. 2000;21:109-114.

11. Mori M, Hariharan M, Anandakumar M, et al. A case-control study on risk factors for pancreatic diseases in Kerala, India. Hepato-Gastroenterology. 1999;46:25-30.

12. Morton C, Klatsky AL, Udaltsova N. Smoking, coffee, and pancreatitis. Am J Gastroenterol. 2004;99:731-738.

13. Nakamura Y, Kobayashi Y, Ishikawa A, et al. Severe chronic pancreatitis and severe liver cirrhosis have different frequencies and are independent risk factors in male Japanese alcoholics. J Gastroenterol. 2004;39:879-887.

14. Rothenbacher D, Lőw M, Hardt PD, et al. Prevalence and determinants of exocrine pancreatic insufficiency among older adults: results of a population-based study. Scand J Gastroenterol. 2005;40:697-704.

15. Talamini G, Bassi C, Falconi M, et al. Cigarette smoking: an independent risk factor in alcoholic pancreatitis. Pancreas. 1996;2:131-137.

16. Talamini G, Bassi C, Falconi M, et al. Alcohol and smoking as risk factors in chronic pancreatitis and pancreatic cancer. Dig Dis Sci. 1999;44:1303-1311.
17. Talamini G, Vaona B, Bassi C, et al. Alcohol intake, cigarette smoking, and body mass index in patients with alcohol-associated pancreatitis. J Clin Gastroenterol. 2000;31:314-317.

18. Yadav D, Eigenbrodt ML, Briggs MJ, et al. Pancreatitis: prevalence and risk factors among male veterans in a detoxification program. Pancreas. 2007;34:390-398.

19. Tolstrup JS, Kristiansen L, Becker U, et al. Smoking and risk of acute and chronic pancreatitis among women and men: a population-based cohort study. Arch Intern Med. 2009;169:603-609.

20. Yadav D, Hawes RH, Brand RE, et al. North American Pancreatic Study Group. Alcohol consumption, cigarette smoking, and the risk of recurrent acute and chronic pancreatitis. Arch Intern Med. 2009;169:1035-1045.

21. Room R. Smoking and drinking as complementary behaviours. Biomed Pharmacother. 2004;58:111-115.

22. Bobo JK, Husten C. Sociocultural influences on smoking and drinking. Alcohol Res Health. 2000;24:225-232.

23. Anthony JC, Echeagaray-Wagner F. Epidemiologic analysis of alcohol and tobacco use-pattern of co-occurring consumption and dependence in the United States. Alcohol Res Health. 2000;24:201-208.

24. Maisonneuve P, Lowenfels AB, Müllhaupt B, et al. Cigarette smoking accelerates progression of alcoholic chronic pancreatitis. Gut. 2005;54:510-514.

25. Apte MV, Pirola RC, Wilson JS. Where there's smoke there's not necessarily fire. Gut. 2005;54:446-447.

26. Greenland S. Quantitative methods in the review of epidemiologic literature. Epidemiol Rev. 1987;9:1-30.

27. van Houwelingen HC, Arends LR, Stijnen T. Advanced methods in meta-analysis: multivariate approach and meta-regression. Stat Med. 2002;21:589-624.

28. Higgins JP, Thompson SG. Quantifying heterogeneity in a meta-analysis. Stat Med. 2002;21:1539-1558.

29. Egger M, Davey SG, Schneider M, et al. Bias in meta-analysis detected by a simple, graphical test. BMJ. 1997;315:629-634.

30. Lévy P, Barthet M, Mollard BR, et al. Estimation of the prevalence and incidence of chronic pancreatitis and its complications. Gastroenterol Clin Biol. 2006;30:838-844.

31. Yang AL, Vadhavkar S, Singh G, et al. Epidemiology of alcohol-related liver and pancreatic disease in the United States. Arch Intern Med. 2008;168:649-656.

32. Gandini $\mathrm{S}$, Botteri $\mathrm{E}$, Iodice $\mathrm{S}$, et al. Tobacco smoking and cancer: a meta-analysis. Int J Cancer. 2008;122:155-164.

33. Bueno de Mesquita HB, Maisonneuve P, Moerman CJ, et al. Life-time history of smoking and exocrine carcinoma of the pancreas: a population-based case-control study in the Netherlands. Int $J$ Cancer. 1991;49:816-822.

34. Lindkvist B, Appelros S, Manjer J, et al. A prospective cohort study of smoking in acute pancreatitis. Pancreatology. 2008;8:63-70.

35. Pelli H, Lappalainen-Lehto R, Piironen A, et al. Risk factors for recurrent acute alcohol-associated pancreatitis: a prospective analysis. Scand J Gastroenterol. 2008;43:614-621.

36. Imoto M, DiMagno EP. Cigarette smoking increases the risk of pancreatic calcification in late-onset but not early-onset idiopathic chronic pancreatitis. Pancreas. 2000;21:115-119.

37. Maisonneuve P, Frulloni L, Müllhaupt B, et al. Impact of smoking on patients with idiopathic chronic pancreatitis. Pancreas. 2006;33:163-168.

38. Lowenfels AB, Maisonneuve P, Whitcomb DC, et al. Cigarette smoking as a risk factor for pancreatic cancer in patients with hereditary pancreatitis. JAMA. 2001;286:169-170.

39. Howes N, Greenhalf W, Neoptolemos J. Screening for early pancreatic ductal adenocarcinoma in hereditary pancreatitis. Med Clin North Am. 2000;84:719-738. 